\title{
Síntese e caracterização de hidróxidos duplos a partir da lama vermelha
}

\section{(Synthesis and characterization of layered double hydroxides from red mud)}

\author{
M. V. P. O. Cunha, J. A. M. Corrêa \\ Instituto de Geociências, Universidade Federal do Pará, Av. Augusto Corrêa s/n, Belém, PA 66093-110
}

\begin{abstract}
Resumo
O resíduo do refino da bauxita, comumente conhecido como lama vermelha, tem mostrado ser capaz de ligar metais traços tais como o cobre, cádmio, zinco, cromo, níquel e chumbo sob condições apropriadas. A neutralização da lama vermelha é necessária porque essa lama é tipicamente cáustica, com pH em torno de 13. Muitas pesquisas têm sido desenvolvidas envolvendo a utilização da lama vermelha, porque contem uma série de elementos valiosos. Assim a utilização da lama vermelha como material de construção e como adsorvente é atrativa porque ela é utilizada em grande quantidade. Nada pode ser feito sem descartar componentes valiosos como titânio, zircônio e principalmente ferro. A síntese de hidrotalcita conectada com a reciclagem de soluções alcalinas representa uma maneira efetiva de uso dessa solução considerando a forte demanda por proteção ambiental no mundo. Os compostos tipo hidrotalcita, precipitados durante a neutralização da lama, também removem oxi-ânions de metais de transição através da combinação de intercalações e adsorção de espécies aniônicas na sua superfície externa. Os hidróxidos duplos lamelares (HDL) vêm sendo investigados à muitos anos como materiais hospedeiros para uma variedade de reações de intercalação de anions. A estrutura lamelar dos HDLs pode ser usada para controlar a adição ou remoção de uma variedade de espécies químicas, tanto orgânicas quanto inorgânicas. Isto é alcançado através de sua habilidade de adaptar a separação das lamelas de hidróxidos, e da reatividade da região interlamelar. O material resultante adsorve anions quando colocado em soluções e reverte a estrutura da hidrotalcita. Avanços significativos têm sido alcançados recentemente na caracterização desses materiais, incluindo estudos estruturais sobre o mecanismo de intercalação. O objetivo desse trabalho foi a síntese e caracterização de HDLs a partir da lama vermelha, e testar esses materiais como adsorventes de metais pesados.
\end{abstract}

Palavras-chave: lama vermelha, hidróxidos duplos lamelares, síntese.

\begin{abstract}
Bauxite refinery residues, commonly known as "red mud", have been shown to be capable of binding trace metals such as copper, cadmium, zinc, chromium, nickel and lead under suitable conditions. Neutralization of red mud is necessary because red muds are typically highly caustic, with a reaction $\mathrm{pH}$ of about 13. Much research has been carried out on utilization of red mud because it contains a lot of valuable components. Although utilization of the red mud as building material and as an absorbent is attractive because the red mud is used in bulk, nothing can be done without discarding valuable components such as titanium, zirconium and the main iron. Hydrotalcite-synthesis connected with recycling of alkali-solution is a prospective way for effective use of the solution under the strong demand for the environmental protection in the world. The hydrotalcite-like compounds, precipitated during neutralization, also remove oxy-anions of transition metals through a combination of intercalation and adsorption of the anionic species on the external surfaces. Layered double hydroxides (LDHs) have been investigated for many years as host materials for a range of anion exchange intercalation reactions. The lamellar structure of LDHs can be used for the controlled addition or removal of a variety of species, both organic and inorganic. This is achieved through their ability to adjust the separation of the hydroxide layers, and the reactivity of the interlayer region. The resultant material adsorbs anions when placed in solution and reverts to the hydrotalcite structure. Significant advances have been made recently on the characterization of these materials, including structural studies on the mechanism of intercalation. The aim of this work was the synthesis and characterizations of LDHs from red mud that will be tested as adsorbent material for heavy metals.
\end{abstract}

Keywords: red mud, layered double hydroxides (LDHs), synthesis.

\section{INTRODUÇÃO}

Os hidróxidos duplos lamelares (HDL) são compostos que apresentam dois cátions metálicos na lamela e espécies aniônicas no domínio interlamelar neutralizando as cargas. São também chamados de compostos do tipo hidrotalcita ou argilas aniônicas [1]. Estes compostos têm formula geral: $\mathrm{M}_{\mathrm{a}}(\mathrm{II}) \mathrm{M}_{\mathrm{b}}(\mathrm{III})(\mathrm{OH})_{2 \mathrm{a}+2 \mathrm{~b}}(\mathrm{X}-)_{b} \cdot \mathrm{nH}_{2} \mathrm{O}$ onde $\mathrm{M}(\mathrm{II})$ é um metal divalente, $\mathrm{M}(\mathrm{III}) \mathrm{um}$ metal trivalente e $\mathrm{X}$ um ânion intercalado. Os ânions e as moléculas de água localizados na região interlamelar são arranjados de maneira desordenada, sendo livres para se moverem através da quebra e formação de ligações com as lamelas [2]. A mobilidade dos ânions no domínio interlamelar propicia a substituição destes por troca iônica em solução, sendo que um grande número de ânions, tanto orgânicos como inorgânicos, pode ocupar este domínio [1]. Os HDLs, naturais ou sintéticos, contendo vários cátions metálicos vêm sendo sintetizados e estudados 
por diversos pesquisadores. Os cátions divalentes mais comum são o $\mathrm{Mg}, \mathrm{Mn}, \mathrm{Fe}, \mathrm{Co}, \mathrm{Ni}, \mathrm{Cu}, \mathrm{Zn}$ e $\mathrm{Ca}$; enquanto que os cátions trivalentes mais comuns são o $\mathrm{Al}, \mathrm{Cr}, \mathrm{Mn}$, $\mathrm{Fe}, \mathrm{Co}, \mathrm{Ni}$ [3]. Muitos autores utilizam várias combinações destes cátions di e trivalentes na síntese de HDLs, obtendo resultados variados, dependendo da composição e do método de síntese utilizado [4-6]. Dependendo de sua composição, cristalinidade, estabilidade térmica e outras propriedades físico-químicas, os HDLs podem apresentar uma grande variedade de aplicações. Entre as aplicações, sugere-se na literatura a síntese de HDLs a partir da lama vermelha, com adição de substâncias ou soluções enriquecidas em metais bivalentes [7].

A lama vermelha representa um passivo ambiental importante para a indústria de beneficiamento de alumínio, devido aos riscos de contaminação do meio ambiente e aos custos associados ao seu manejo e disposição. Em conseqüência, a Comissão Mundial sobre Meio Ambiente e Desenvolvimento cita entre as atividades industriais mais poluentes a indústria de metais não-ferrosos, e, dentre estes, a do alumínio [8]. O Brasil é um dos maiores produtores de bauxita, ocupando lugar de destaque no cenário mundial. A produção nacional de bauxita chegou recentemente à cerca de 13 milhões de toneladas/ano, colocando o Brasil entre os quatro principais produtores. No Estado do Pará encontram-se as principais reservas nacionais, situadas nos municípios de Oriximiná, Almeirim, Juruti, Faro, Paragominas e S. Domingos do Capim. De acordo com a Associação Brasileira do Alumínio as principais empresas que trabalham com a extração da bauxita são: Mineração Rio do Norte S.A. (Porto Trombetas, Oriximiná, Pará), Companhia Brasileira de Alumínio, Alumínio S.A., Alumínio do Brasil S.A. [9]. No município de Barcarena está localizada a Alunorte, que beneficia a bauxita extraída pela MRN. Atualmente, a Alunorte caminha para se tornar a maior refinaria de alumina do mundo. Tem produção de 4,4 milhões de toneladas anuais, devendo ampliar a produção para 6,3 milhões de toneladas até 2008 [10]. A disposição não adequada da lama vermelha pode acarretar em problemas como: a) contaminação da água de superfície e subterrânea por $\mathrm{NaOH}$, ferro, alumínio ou outro agente químico; b) contato direto com animais, plantas e seres humanos; c) o vento pode carrear pó dos depósitos de lama vermelha seca, formando nuvens de poeira alcalina; d) impacto visual sobre uma extensa área. Alguns casos de acidentes ambientais provocados pela disposição inadequada da lama vermelha em vários países são relatados na literatura. Assim, a crescente preocupação ambiental com a disposição aceitável de resíduos tóxicos, tais como a lama vermelha, gerou um número maior de pesquisas em torno da utilização deste resíduo. As principais aplicações resumem-se em: utilização como matéria prima na indústria de cimento, fabricação de materiais de construção, fabricação de ladrilhos cerâmicos e recuperação de metais de valor [11]. Além disso, a lama vermelha tem o potencial de diminuir a acidez de águas de drenagem de minas, águas contaminadas e solos contaminados por metais pesados.
Isso ocorre pelo fato da lama vermelha ser fortemente alcalina [12]. Na recuperação de áreas degradadas a lama vermelha é utilizada na remediação de áreas contaminadas e no tratamento de efluentes líquidos, tendo sido utilizada com sucesso no tratamento de águas ácidas de minas [13, 14]. A lama vermelha também é utilizada na remediação de solos contaminados por metais pesados [15]. Na indústria química a lama vermelha tem sido utilizada como catalisador em várias aplicações como, por exemplo, na degradação de compostos orgânicos voláteis (COV) e na degradação de compostos organoclorados [16, 17]. As propriedades de adsorção da lama vermelha são aproveitadas no tratamento de efluentes, tendo sido aplicada em processos de adsorção para vários tipos de adsorbatos, em efluentes sintéticos e reais, como metais pesados $\mathrm{Cu}(\mathrm{II}), \mathrm{Pb}(\mathrm{II}), \mathrm{Cd}$ (II), $\mathrm{Cr}(\mathrm{VI})$ e ânions como fosfato $\left(\mathrm{PO}_{4}{ }^{3-}\right)$ e pigmentos têxteis [18-27]. No tratamento de esgotos domésticos, a lama tem sido utilizada tanto para a remoção de fósforo e formas de nitrogênio $\left(\mathrm{NH}_{4}^{-} \mathrm{e} \mathrm{NO}_{3}^{-}\right)$como para a remoção de vírus e bactérias [28, 29]. Na literatura é reportada a utilização da lama vermelha para a adsorção e clarificação de chorume, obtendo uma redução na carga orgânica de aproximadamente $60 \%$ de remoção de cor [30, 31]. Produtos desenvolvidos a partir da lama vermelha para o tratamento de matrizes ambientais receberam denominação comercial e alguns foram patenteados como, por exemplo, a Kaiser Aluminium \& Chem. Co. que desenvolveu um

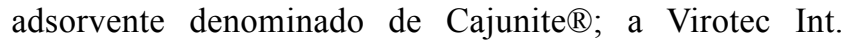
Ltd. que desenvolveu um processo em que se obtém um adsorvente a partir da lama vermelha neutralizada com água do mar, que patenteou como Basecon ${ }^{\circledR}$ e o produto obtido como Bauxsol6 [26, 32, 33]. A indústria Alcoa, juntamente com o Departamento de Agricultura do Oeste da Austrália, desenvolveu o Alkaloam $\AA$, para uso agrícola [34]. Além desses usos, a lama vermelha neutralizada pode ser utilizada como material de partida para a produção de vários produtos, entre eles os HDLs, que por sua vez, podem ser aplicados em processos de remediação ambiental. Assim, o objetivo desse estudo é compreender que fatores controlam a síntese de HDLs diretamente a partir da lama vermelha para posteriormente testar esse material como adsorvente de metais pesados. A escolha do tema levou em consideração as adversidades decorrentes dos processos de síntese dos HDLs a partir da lama vermelha, e, ao fato desse resíduo apresentar cátions trivalentes e tetravalentes que podem servir como matéria prima para a produção dos HDLs por adição de compostos contendo elementos bivalentes. Serão apresentados os resultados da síntese de três experimentos, no qual foi variado o tempo de gotejamento e tempo de banho hidrotérmico.

\section{EXPERIMENTAL}

A lama vermelha utilizada nesse trabalho foi coletada após a digestão industrial da bauxita da planta industrial da Alunorte, PA. A preparação dos HDLs utilizados seguiu os métodos da co-precipitação a pH variável (decrescente) 
Tabela I - Identificação dos compostos sintetizados e condições de sínteses.

[Table I - Identification of the compounds synthesized and conditions of synthesis.]

\begin{tabular}{|c|c|c|c|c|c|c|}
\hline $\mathrm{E}^{*}$ & $\begin{array}{l}\text { Compostos } \\
\text { sintetizados }\end{array}$ & $\begin{array}{l}\text { Tempo de } \\
\text { gotejamento } \\
\text { (h) }\end{array}$ & $\begin{array}{c}\text { Tempo } \\
\text { do banho } \\
\text { hidrotérmico } \\
\text { (dia) }\end{array}$ & $\begin{array}{l}\text { Temperatura } \\
\text { do banho } \\
\text { hidrotérmico } \\
\left({ }^{\circ} \mathrm{C}\right) \\
\end{array}$ & $\begin{array}{l}\text { Razão } \\
\text { Molar } \\
\text { (teórica) }\end{array}$ & $\mathrm{pH}$ \\
\hline \multirow{5}{*}{1} & HDL-2 & 7 & 5 & 80 & $\mathrm{R}: 2 ; \mathrm{x}: 0,33$ & 10 \\
\hline & HDL-10 & 7 & 10 & 80 & $\mathrm{R}: 2 ; \mathrm{x}: 0,33$ & 11 \\
\hline & HDL-3 & 7 & 20 & 80 & $\mathrm{R}: 2 ; \mathrm{x}: 0,33$ & 11 \\
\hline & HDL-13 & 7 & 30 & 80 & $\mathrm{R}: 2 ; \mathrm{x}: 0,33$ & 10 \\
\hline & HDL-11 & 7 & 60 & 80 & $\mathrm{R}: 2 ; \mathrm{x}: 0,33$ & 9 \\
\hline \multirow{5}{*}{2} & HDL-18 & 5 & 5 & 80 & $\mathrm{R}: 2 ; \mathrm{x}: 0,33$ & 12 \\
\hline & HDL-16 & 5 & 10 & 80 & $\mathrm{R}: 2 ; \mathrm{x}: 0,33$ & 11 \\
\hline & HDL-15 & 5 & 20 & 80 & $\mathrm{R}: 2 ; \mathrm{x}: 0,33$ & 10 \\
\hline & HDL-14 & 5 & 30 & 80 & $\mathrm{R}: 2 ; \mathrm{x}: 0,33$ & 12 \\
\hline & HDL-12 & 5 & 60 & 80 & $\mathrm{R}: 2 ; \mathrm{x}: 0,33$ & 10 \\
\hline \multirow{5}{*}{3} & HDL-23 & 3 & 5 & 80 & $\mathrm{R}: 2 ; \mathrm{x}: 0,33$ & 9 \\
\hline & HDL-22 & 3 & 10 & 80 & $\mathrm{R}: 2 ; \mathrm{x}: 0,33$ & 11 \\
\hline & HDL-21 & 3 & 20 & 80 & $\mathrm{R}: 2 ; \mathrm{x}: 0,33$ & 11 \\
\hline & HDL-20 & 3 & 30 & 80 & $\mathrm{R}: 2 ; \mathrm{x}: 0,33$ & 13 \\
\hline & HDL-19 & 3 & 60 & 80 & $\mathrm{R}: 2 ; \mathrm{x}: 0,33$ & 13 \\
\hline \multirow{5}{*}{4} & HDL-25 & 5 & 5 & $\mathrm{TA}^{*}$ & $\mathrm{R}: 2 ; \mathrm{x}: 0,33$ & 9 \\
\hline & HDL-28 & 5 & 5 & 40 & $\mathrm{R}: 2 ; \mathrm{x}: 0,33$ & 9 \\
\hline & HDL-26 & 5 & 5 & 60 & $\mathrm{R}: 2 ; \mathrm{x}: 0,33$ & 9 \\
\hline & HDL-18 & 5 & 5 & 80 & $\mathrm{R}: 2 ; \mathrm{x}: 0,33$ & 12 \\
\hline & HDL-27 & 5 & 5 & 100 & $\mathrm{R}: 2 ; \mathrm{x}: 0,33$ & 10 \\
\hline \multirow{6}{*}{5} & HDL-30 & 5 & 5 & 80 & $\mathrm{R}: 1,5 ; \mathrm{x}: 0,4$ & 13 \\
\hline & HDL-18 & 5 & 5 & 80 & $\mathrm{R}: 2 ; \mathrm{x}: 0,33$ & 12 \\
\hline & HDL-31 & 5 & 5 & 80 & $\mathrm{R}: 2,3 ; \mathrm{x}: 0,3$ & 13 \\
\hline & HDL-33 & 5 & 5 & 80 & $\mathrm{R}: 3 ; \mathrm{x}: 0,25$ & 13 \\
\hline & HDL-32 & 5 & 5 & 80 & $\mathrm{R}: 4 ; \mathrm{x}: 0,2$ & 13 \\
\hline & HDL-34 & 5 & 5 & 80 & $\mathrm{R}: 2 ; \mathrm{x}: 0,33$ & 7 \\
\hline \multirow{4}{*}{6} & HDL-35 & 5 & 5 & 80 & $\mathrm{R}: 2 ; \mathrm{x}: 0,33$ & 8 \\
\hline & HDL-37 & 5 & 5 & 80 & $\mathrm{R}: 2 ; \mathrm{x}: 0,33$ & 9 \\
\hline & HDL-36 & 5 & 5 & 80 & $\mathrm{R}: 2 ; \mathrm{x}: 0,33$ & 10 \\
\hline & HDL-38 & 5 & 5 & 80 & $\mathrm{R}: 2 ; \mathrm{x}: 0,33$ & 11 \\
\hline
\end{tabular}

*TA: Temperatura Ambiente; E*: Experimentos

[4], e pH constante [1]. Os HDLs foram sintetizados a partir de soluções de hidróxido de sódio, soluções de cloreto de magnésio hexa-hidratado e lama vermelha dissolvida em ácido clorídrico, atendendo a estequiometria dos hidróxidos duplos lamelares, com a razão $\mathrm{x}\left(\mathrm{M}^{3+} / \mathrm{M}^{3+}+\mathrm{M}^{2+}\right)$ variando de 0,2 a 0,4 e a razão molar $\mathrm{R}\left(\mathrm{M}^{2+} / \mathrm{M}^{3+}\right)$ variando de 4,0 a 1,5. Após a formação do precipitado e término do gotejamento, os compostos foram submetidos a um tratamento hidrotérmico em diferentes temperaturas para avaliar seu efeito sobre a formação e cristalinidade dos compostos. Em seguida os compostos foram resfriados à temperatura ambiente, lavados com grande quantidade de água deionizada e levados a estufa para secagem. Os HDLs secos foram então caracterizados por difração de raios $\mathrm{X}$ (método do pó), espectroscopia na faixa do infravermelho (IV), análise térmica (ATD/TG) e microscopia eletrônica de varredura (MEV). O trabalho foi dividido em seis experimentos, no qual foi variado o tempo de gotejamento ( 3,5 e $7 \mathrm{~h})$ e o tempo de banho hidrotérmico $(5,10,20$, 30 e 60 dias) nos experimentos 1,2 e 3 ; a temperatura do banho hidrotérmico no experimento 4; a razão molar no experimento 5; o pH no experimento 6 . Na Tabela I são mostradas a identificação dos compostos sintetizados e as condições de sínteses. 


\section{Caracterização dos materiais}

Inicialmente foi feita a caracterização química e mineralógica da lama vermelha. A caracterização mineralógica foi feita por difração de raios X. Para determinar a concentração dos elementos importantes para a síntese foram seguidos métodos químicos clássicos. A concentração do alumínio e do ferro foi determinada por titrimetria e a de magnésio por espectrometria de absorção atômica, PerkinElmer 3300, com comprimento de onda 285,2 nm. O gás de ionização utilizado foi acetileno. As fases mineralógicas nos compostos sintetizados foram determinadas por difração de raios $\mathrm{X}$, método do pó, em amostra total em um difratômetro de raios X PANalytical X'Pert com goniômetro PW 3050/60 (teta/teta), modelo PW3373/00, foco fino longo, $2200 \mathrm{~W}, 60$ $\mathrm{kV}$ e com tubo de raios $\mathrm{X}$ cerâmico de anodo de Co $(\mathrm{k} \alpha 1$ $1,78901 \AA$ ). A aquisição de dados foi de 5 a $80^{\circ} 2 \theta$.

As medidas de espectroscopia na região do infravermelho foram processadas em um espectrofotômetro Perkin Elmer 1760, com registros da faixa espectral de 4000 a 400 $\mathrm{cm}^{-1}$, com resolução de $4 \mathrm{~cm}^{-1}$. Para a análise as amostras foram preparadas a partir de misturas de $0,0015 \mathrm{~g}$ do composto sintetizado com $0,2 \mathrm{~g}$ de brometo de potássio. Essas misturas foram trituradas e prensadas sob pressão de 1,8 kbar, obtendo-se assim pastilhas translúcidas.

A análise termogravimétrica foi obtida no termoanalisador Stanton Redcroft, equipado com um programador de temperatura e uma microbalança, com forno cilíndrico vertical e com conversor digital acoplado a um microcomputador. Foi utilizado um cadinho de porcelana, massa de amostra entre 10 a $20 \mathrm{mg}$, taxa de aquecimento 5 ${ }^{\circ} \mathrm{C} /$ min, temperatura máxima $1100^{\circ} \mathrm{C}$.

A concentração do alumínio foi determinada por titrimetria. A concentração de magnésio e ferro foi determinada por espectrometria de absorção atômica, Perkin Elmer 3300, com comprimento de onda $285,2 \mathrm{~nm}$ para o magnésio e $\lambda=248,3 \mathrm{~nm}$ para o ferro. $\mathrm{O}$ gás de ionização utilizado foi $\mathrm{o}$ acetileno.

A determinação da quantidade da água de hidratação foi determinada por análise termogravimétrica.

As imagens por microscopia eletrônica de varredura foram obtidas em um equipamento LEO-1430. As condições de análises para imagens de elétrons secundários foram: corrente do feixe de elétrons $90 \mu \mathrm{A}$, voltagem 10 $\mathrm{kV}$ e distância de trabalho $12-15 \mathrm{~mm}$. As amostras foram metalizadas com uma liga de ouro e platina. Concomitante às análises de microscopia, foi determinada a proporção de cátions metálicos $\mathrm{M}(\mathrm{II}): \mathrm{M}$ (III) por meio da análise de espectroscopia de dispersão de energia de raios $\mathrm{X}$.

\section{RESULTADOS E DISCUSSÃO}

Os principais constituintes mineralógicos da lama vermelha utilizada neste trabalho foram gibbsita, hematita, anatásio, goethita, sodalita. A concentração dos elementos mais abundantes mostra os teores de $\mathrm{Al}_{2} \mathrm{O}_{3}(23,05 \%), \mathrm{Fe}_{2} \mathrm{O}_{3}$ (36,35\%), $\mathrm{TiO}_{2}(3,45 \%), \mathrm{SiO}_{2}(13,78 \%), \mathrm{Na}_{2} \mathrm{O}(11,20 \%) \mathrm{e}$ perda ao fogo (10,19\%). Como a amostra de lama vermelha apresentou aproximadamente $36 \% \mathrm{Fe}$, na análise de DRX foi necessário mudar a janela do discriminador para diminuir o background proveniente da fluorescência do Fe. A indexação dos picos basais nos difratogramas obtidos a partir dos materiais preparados foi realizada por comparação com o padrão do banco de dados do programa Philips X-Pert High Score, utilizando os padrões JCPDS-ICDD. O padrão utilizado foi a hidrotalcita $\left[\mathrm{Mg}-\mathrm{Al}-\mathrm{CO}_{3}\right]$, arquivo 89-0460, HDL catalogado nesse banco de dados. Após a indexação dos picos, utilizou-se a equação de Bragg para determinar os espaçamentos basais, que foram calculados pela média das

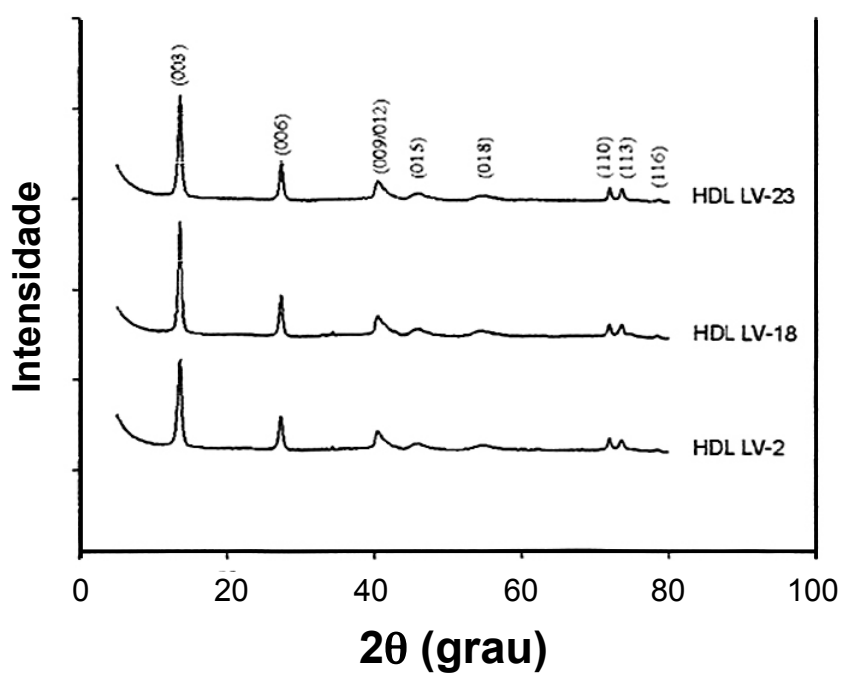

Figura 1: Difratogramas de raios $\mathrm{X}$ dos compostos sintetizados no experimento 1 (HDL LV-2), experimento 2 (HDL LV-18) e experimento 3 (HDL LV-23).

[Figure 1: X-ray diffraction patterns of compounds of experiment 1 (HDL LV-2), experiment 2 (HDL LV-18) and experiment 3 (HDL $L V-23)$.

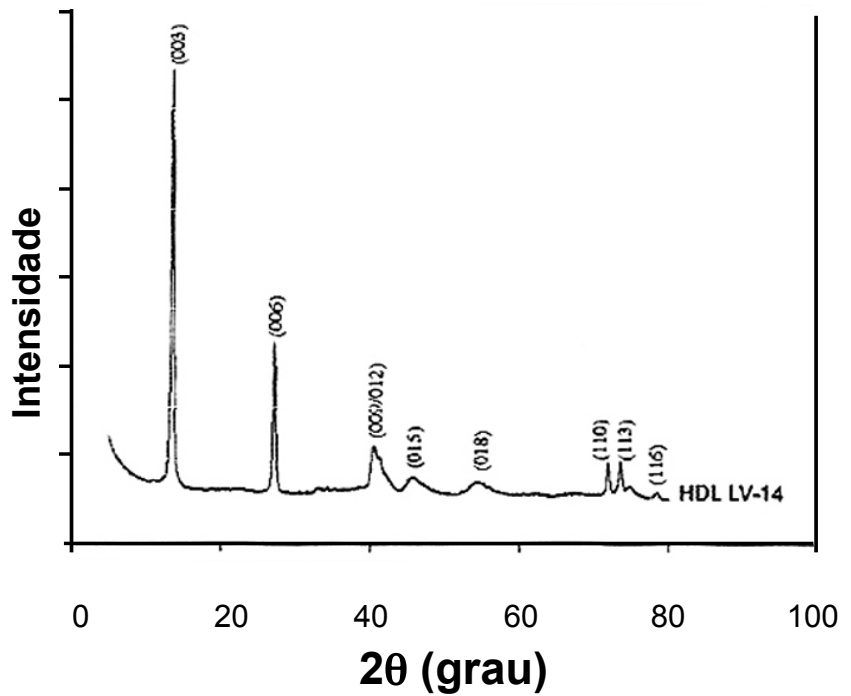

Figura 2: Difratogramas de raios $\mathrm{X}$ do composto sintetizado no experimento 2 (HDL LV-14).

[Figure 2: X-ray diffraction patterns of compounds of experiment 2 (HDL LV-14).] 
distâncias encontradas para os picos basais identificáveis e de boa definição.

Nas Figs. 1 e 2 são mostrados os resultados das análises de difração de raios $\mathrm{X}$ dos compostos sintetizados nos experimentos 1,2 e 3, para o tempo de banho hidrotérmico de 5 dias e tempo de gotejamento de $7 \mathrm{~h}, 5 \mathrm{~h} \mathrm{e} 3 \mathrm{~h}$, respectivamente. Os difratogramas mostram que os compostos podem ser caracterizados como lamelares devido à repetição dos picos basais (003, 006, 009, 012, 015, 018, 110 e 113). Por meio dos valores obtidos dos picos de índice 003 e 006 , foi possível calcular o espaçamento basal médio 7,62 A. Este valor é característico para HDLs com intercalação do ânion carbonato [35].

Os compostos que apresentaram maior cristalinidade foram os submetidos a tempo de banho hidrotérmico de 30 e 60 dias. Alguns difratogramas indicaram a presença de outra fase mineral, identificada como hematita (JCPDSICDD 24-0072). A presença de hematita ocorreu somente nos compostos que foram submetidos a um maior tempo de tratamento hidrotérmico, o que sugere que está condição facilita sua formação (Fig. 3).

Para confirmar os resultados de análise de proporção entre os cátions, nos HDLs preparados, foram realizadas análises pela técnica de EDX, sendo as razões entre os cátions de aproximadamente 1,5, 2, 2,33, 3 e 4. Comparando estas razões com as obtidas por métodos clássicos ( $\mathrm{Fe}$ e $\mathrm{Al}$ - colorimetria, $\mathrm{Mg}$ - espectrometria de absorção atômica), podemos considerar que houve boa concordância, já que se tratam de técnicas diferentes. Entretanto, deve-se considerar que a técnica de EDX é menos precisa porque, os cátions $\mathrm{Mg}^{2+}$ ou $\mathrm{Al}^{3+}$ apresentam picos de emissão muito próximos e podem, por isso interferir no resultado da análise. $\mathrm{O}$ melhor resultado de síntese foi obtido com razão 4 e pH 9. Nesta condição não houve formação de fase paralela (Fig. 4).

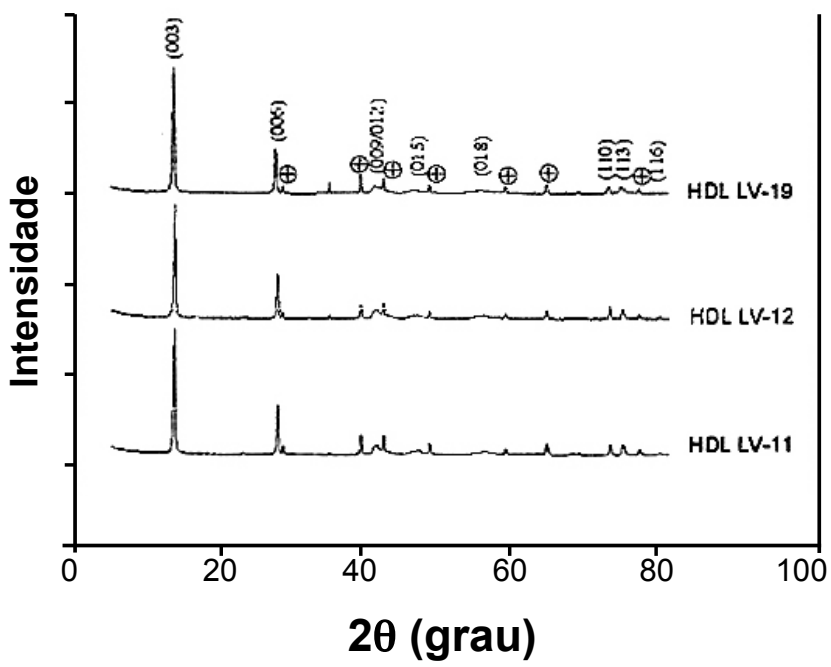

Figura 3: Difratogramas de raios $\mathrm{X}$ dos compostos sintetizados no experimento 1 (HDL LV-11), experimento 2 (HDL LV-12), experimento 3 (HDL LV-19) e $\bigoplus$ hematita.

[Figure 3: X-ray diffraction patterns of compounds of experiment 1 (HDL LV-11), experiment 2 (HDL LV-12), experiment 3 (HDL $L V$-19) and $\bigoplus$ hematite.]

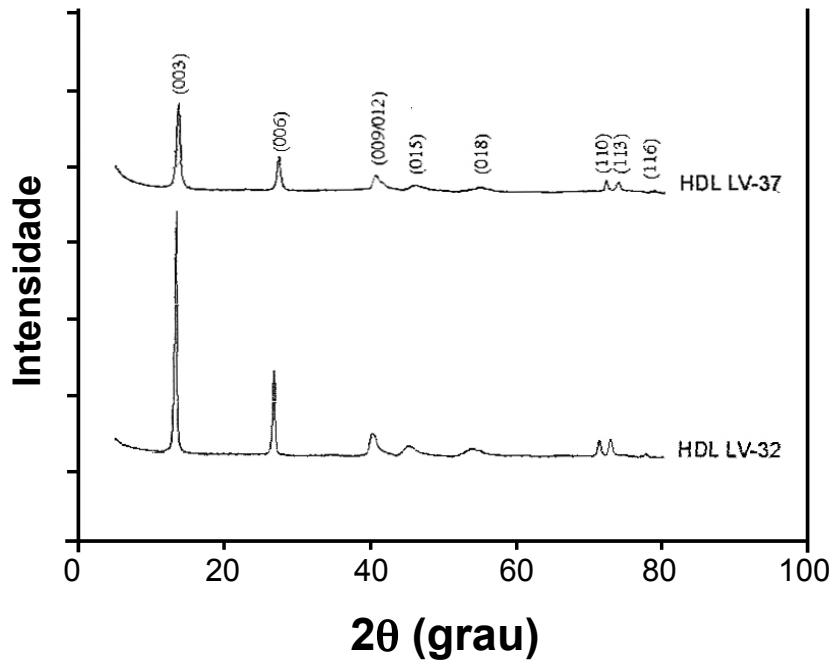

Figura 4: Difratogramas de raios $\mathrm{X}$ dos compostos sintetizados a pH 9 (HDL LV-37) e razão 4 (HDL LV-32).

[Figure 4: X-ray diffraction patterns of compounds synthesized at pH 9 (HDL LV-37) and ratio 4 (HDL LV-32).]

Os valores dos parâmetros da cela unitária a e c foram calculados pelo método dos mínimos quadrados a partir das reflexões dos difratogramas dos compostos sintetizados. $\mathrm{O}$ sistema cristalino dos HDLs sintetizados é o romboédrico com grupo espacial R-3m. Na Tabela II são mostradas a identificação dos compostos sintetizados, os valores obtidos dos parâmetros a e c da célula unitária e os valores da distância interplanar (d).

Os resultados da análise de espectroscopia na região do infravermelho dos compostos sintetizados são apresentadas nas Fig. 5 (experimento 1), Fig. 6 (experimento 2) e Fig. 7 (experimento 3). Os espectros foram semelhantes em todos os experimentos. Todos os compostos sintetizados apresentaram uma banda larga e intensa em torno de $3440 \mathrm{~cm}^{-1}$, que é atribuída às vibrações de estiramento de grupos $\mathrm{O}-\mathrm{H}$ referentes a hidroxilas dos HDLs e às moléculas de água presente nos compostos. A banda de menor intensidade, em torno de 1635 $\mathrm{cm}^{-1}$ refere-se à deformação angular das moléculas de água. Além desses, pode se identificar uma banda intensa em torno de $1300 \mathrm{~cm}^{-1}$, atribuída à redução da simetria do carbonato e a desordem natural no espaço interlamelar. As bandas em torno de 785,653 e $554 \mathrm{~cm}^{-1}$ são atribuídas à presença de ligações metal-oxigênio.

As curvas de análise térmica dos compostos sintetizados mostraram resultados no geral muito semelhantes para todos compostos nos três experimentos. Para as amostras analisadas foi possível distinguir três picos endotérmicos das etapas de decomposição. As temperaturas e perdas de massa estão apresentadas na Tabela III. Nas Figs. 8 e 9 são mostradas as curvas das análises térmica diferencial e termogravimétricas dos compostos HDL LV-11, HDL LV-12 e HDL LV-19. Observa-se que a primeira etapa de decomposição corresponde à perda de água interlamelar e de moléculas de água que possivelmente se encontram adsorvidos na superfície do material, essa faixa iniciase na temperatura ambiente até $\sim 272{ }^{\circ} \mathrm{C}$ e corresponde a uma perda de massa de $\sim 14 \%$. A segunda etapa de decomposição de $\sim 272{ }^{\circ} \mathrm{C}$ até cerca de $372{ }^{\circ} \mathrm{C}$ é interpretada como estando 
Tabela II - Identificação dos experimentos, valores dos parâmetros a e c da célula unitária e valores da distância interplanar $\mathbf{d}$ dos compostos sintetizados.

[Table II - Identification of the experiments, values of the parameters $\boldsymbol{a}$ and $\boldsymbol{c}$ unit cell and values of the interplanar distance $\boldsymbol{d}$ of the synthesized compounds.]

\begin{tabular}{|c|c|c|c|c|}
\hline $\mathrm{E}^{*}$ & $\begin{array}{l}\text { Compostos } \\
\text { sintetizados }\end{array}$ & $\mathrm{a}=\mathrm{b}(\AA)$ & c $(\AA)$ & $\mathrm{d}(\AA)$ \\
\hline \multirow{5}{*}{1} & HDL LV-02 & $3,0075 \pm 0,0229$ & $22,0302 \pm 0,6249$ & 7,51 \\
\hline & HDL LV-10 & $3,0118 \pm 0,0067$ & $21,9554 \pm 0,1798$ & 7,65 \\
\hline & HDL LV-03 & $3,0104 \pm 0,0189$ & $22,0415 \pm 0,5158$ & 7,56 \\
\hline & HDL LV-13 & $3,0440 \pm 0,0006$ & $22,6724 \pm 0,0177$ & 7,58 \\
\hline & HDL LV-11 & $3,0446 \pm 0,0002$ & $22,7100 \pm 0,0057$ & 7,56 \\
\hline \multirow{5}{*}{2} & HDL LV-18 & $3,0499 \pm 0,0013$ & $22,7983 \pm 0,0405$ & 7,58 \\
\hline & HDL LV-16 & $3,0438 \pm 0,0008$ & $22,6411 \pm 0,0234$ & 7,59 \\
\hline & HDL LV-15 & $3,0441 \pm 0,0010$ & $22,7042 \pm 0,0317$ & 7,58 \\
\hline & HDL LV-14 & $3,0481 \pm 0,0027$ & $22,8088 \pm 0,0827$ & 7,62 \\
\hline & HDL LV-12 & $3,0457 \pm 0,0008$ & $22,7197 \pm 0,0246$ & 7,56 \\
\hline \multirow{5}{*}{3} & HDL LV-23 & $3,0489 \pm 0,0007$ & $22,8514 \pm 0,0226$ & 7,59 \\
\hline & HDL LV-22 & $3,0455 \pm 0,0006$ & $22,6734 \pm 0,0172$ & 7,58 \\
\hline & HDL LV-21 & $3,0463 \pm 0,0033$ & $22,7683 \pm 0,1020$ & 7,59 \\
\hline & HDL LV-20 & $3,0522 \pm 0,0005$ & $22,9139 \pm 0,0164$ & 7,65 \\
\hline & HDL LV-19 & $3,0489 \pm 0,0007$ & $22,8514 \pm 0,0226$ & 7,60 \\
\hline \multirow{5}{*}{4} & HDL LV-25 & $3,0445 \pm 0,0076$ & $22,7735 \pm 0,1823$ & 7,60 \\
\hline & HDL LV-28 & $3,0382 \pm 0,0032$ & $22,7052 \pm 0,0765$ & 7,66 \\
\hline & HDL LV-26 & $3,0505 \pm 0,0028$ & $22,9317 \pm 0,0897$ & 7,51 \\
\hline & HDL LV-18 & $3,0498 \pm 0,0015$ & $22,7921 \pm 0,0474$ & 7,58 \\
\hline & HDL LV-27 & $3,0446 \pm 0,0002$ & $22,7100 \pm 0,0057$ & 7,58 \\
\hline \multirow{5}{*}{5} & HDL LV-18 & $3,0498 \pm 0,0015$ & $22,7921 \pm 0,0474$ & 7,58 \\
\hline & HDL LV-30 & $3,0604 \pm 0,0153$ & $23,9059 \pm 0,5171$ & 7,72 \\
\hline & HDL LV-31 & $3,0962 \pm 0,0253$ & $24,0440 \pm 0,8707$ & 7,68 \\
\hline & HDL LV-32 & $3,0962 \pm 0,0253$ & $24,0440 \pm 0,8707$ & 7,76 \\
\hline & HDL LV-33 & $3,0624 \pm 0,020$ & $23,0070 \pm 0,0634$ & 7,67 \\
\hline \multirow{4}{*}{6} & HDL LV-35 & $3,0739 \pm 0,0138$ & $23,8337 \pm 0,4740$ & 7,55 \\
\hline & HDL LV-36 & $3,0615 \pm 0,0248$ & $22,8318 \pm 0,7592$ & 7,61 \\
\hline & HDL LV-37 & $3,0433 \pm 0,0019$ & $22,7500 \pm 0,0585$ & 7,60 \\
\hline & HDL LV-38 & $3,0484 \pm 0,0015$ & $22,8569 \pm 0,0455$ & 7,62 \\
\hline
\end{tabular}

$E^{*}$ : experimentos

relacionada à decomposição de parte das hidroxilas presentes nas lamelas. A terceira etapa de decomposição, de $\sim 372{ }^{\circ} \mathrm{C}$ até cerca de $514{ }^{\circ} \mathrm{C}$, está provavelmente relacionada à decomposição do restante das hidroxilas e do ânion carbonato intercalado. A somatória da decomposição das duas últimas etapas correspondem a uma perda de $19 \%$ em massa.

O composto sintetizado foi analisado quimicamente, mostrando a presença dos metais $\mathrm{Al}, \mathrm{Fe}, \mathrm{Mg}$. A partir da fórmula geral dos HDLs, das quantificações dos íons de magnésio, ferro e alumínio e da análise termogravimétrica dos compostos preparados, foi possível determinar a seguinte fórmula mínima: $\mathrm{Mg}_{5,4}(\mathrm{Al}, \mathrm{Fe})_{2,62} \cdot(\mathrm{OH})_{16} \cdot\left(\mathrm{CO}_{3}\right)_{1,37} \cdot \mathrm{YH}_{2} \mathrm{O}$.
As micrografias de microscopia eletrônica de varredura mostram que os compostos sintetizados apresentam dimensões nanométricas $(<0,2 \mu \mathrm{m})$. Em geral são cristalitos com forma hexagonal, formando aglomerados muito similar a texturas observadas em argilominerais. Os compostos que apresentam os cristais mais bem desenvolvidos são aqueles que foram submetidos a um maior tempo de banho hidrotérmico ( 30 e 60 dias). No experimento HDL LV-11 foi realizada uma análise de EDS que mostrou a presença dos elementos magnésio, ferro e alumínio. Na Fig. 10 é apresentada uma imagem representativa de MEV do composto HDL LV-11, onde se pode observar as dimensões nanométricas dos cristalitos e a microporosidade entre eles. 


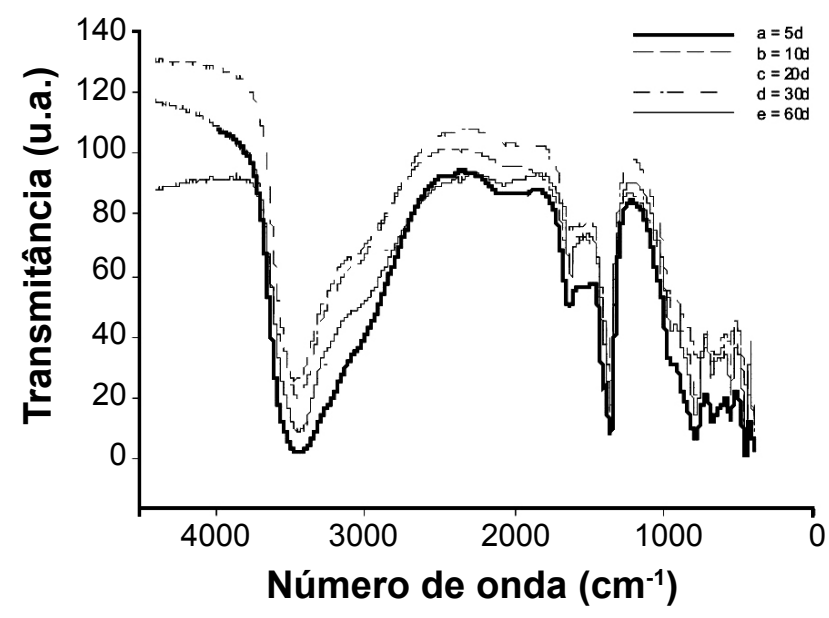

Figura 5: Espectros de infravermelho para os compostos sintetizados no experimento 1: a) HDL LV-2, b) HDL LV-10, c) HDL LV-3, d) HDL LV-13 e e) HDL LV-11.

[Figure 5: Infrared spectra for the compounds synthesized in experiment 1: a) $L V H D L-2$, b) $L V H D L-10$, c) $L V H D L-3$, d) $L V$ $H D L-13$ and e) $L V H D L-11$.]

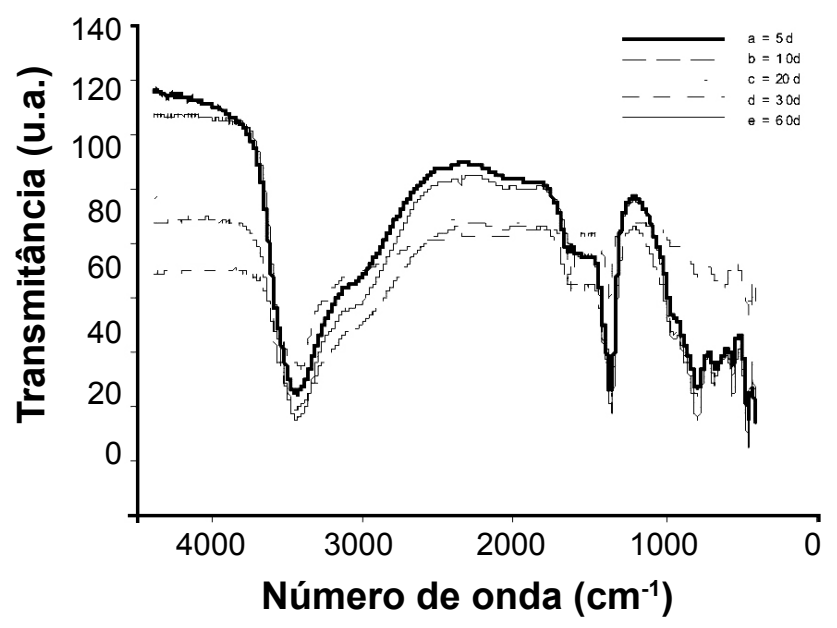

Figura 6: Espectros de infravermelho para os compostos sintetizados no experimento 2: a) HDL LV-18, b) HDL LV-16, c) HDL LV-15, d) HDL LV-14 e e) HDL LV-12.

[Figure 6: Infrared spectra for the compounds synthesized in experiment 2: a) $L V H D L-18, b) L V H D L-16$, c) $L V H D L-15$, d) $L V$ $H D L-14$ and e) $L V H D L-12$.

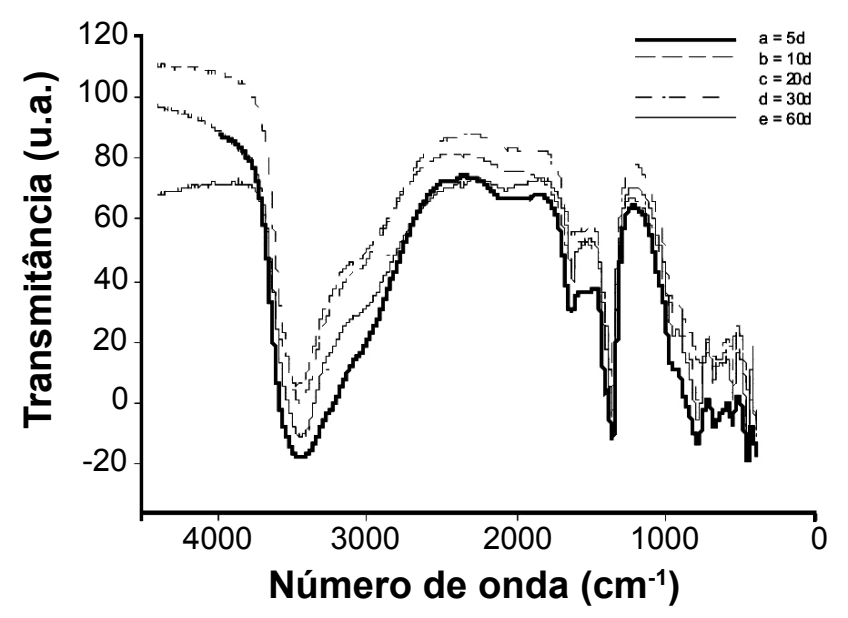

Figura 7: Espectros de infravermelho para os compostos sintetizados no experimento 3: a) HDL LV-23, b) HDL LV-22, c) HDL LV-21, d) HDL LV-20 e e) HDL LV-19.

[Figure 7: Infrared spectra for the compounds synthesized in experiment 3: a) LV HDL-23, b) LV HDL-22, c) LV HDL-21, d) LV HDL-20 and e) LV HDL-19.]

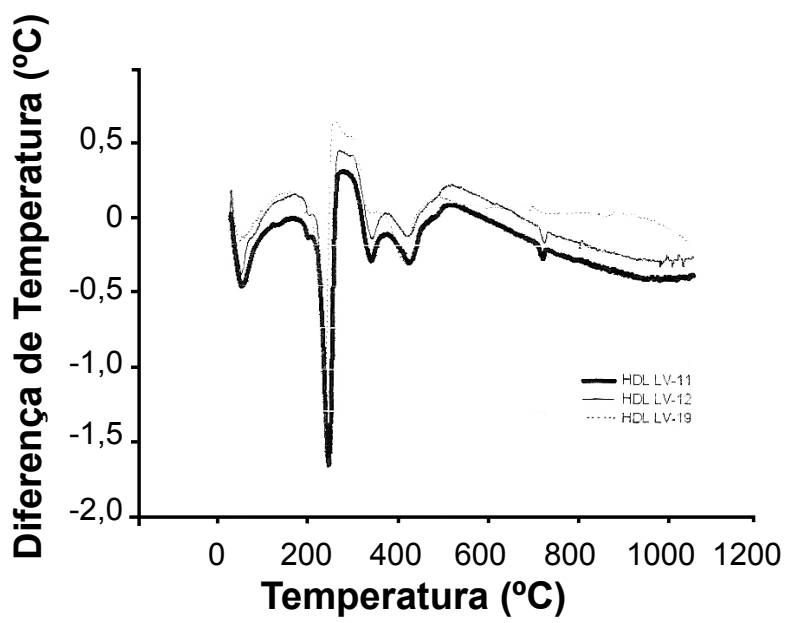

Figura 8: Curvas de análise térmica diferencial das amostras HDL LV-11, HDL LV-12 e HDL LV-19.

[Figure 8: DTA curves of samples HDL-LV 11, LV-12 HDL and HDL LV-19.]

Tabela III - Valores de ATD/TG dos compostos sintetizados.

[Table III - Values of DTA/TGA of the synthesized compounds.]

\begin{tabular}{|c|c|c|c|c|c|c|c|}
\hline \multirow[b]{2}{*}{$\begin{array}{c}\text { Experimento/ } \\
\text { Amostra }\end{array}$} & \multicolumn{2}{|c|}{$1^{\mathrm{a}}$ Etapa } & \multicolumn{2}{|c|}{$2^{\mathrm{a}}$ Etapa } & \multicolumn{2}{|c|}{$3^{\mathrm{a}}$ Etapa } & \multirow[b]{2}{*}{$\begin{array}{c}\text { Massa total } \\
\text { perdida } \\
(\%)\end{array}$} \\
\hline & $\begin{array}{c}\text { Faixa de } \\
\text { Temperatura } \\
\left({ }^{\circ} \mathrm{C}\right)\end{array}$ & $\begin{array}{c}\text { Perda } \\
\text { de } \\
\text { Massa } \\
(\%)\end{array}$ & $\begin{array}{c}\text { Faixa de } \\
\text { Temperatura } \\
\left({ }^{\circ} \mathrm{C}\right)\end{array}$ & $\begin{array}{l}\text { Perda } \\
\text { de } \\
\text { Massa } \\
(\%)\end{array}$ & $\begin{array}{c}\text { Faixa de } \\
\text { Temperatura } \\
\left({ }^{\circ} \mathrm{C}\right)\end{array}$ & $\begin{array}{c}\text { Perda } \\
\text { de } \\
\text { Massa } \\
(\%)\end{array}$ & \\
\hline 1 / HDL LV-11 & TA* - 266 & 14 & $266-370$ & 10 & $370-514$ & 10 & 34 \\
\hline 2 / HDL LV-12 & $\mathrm{TA}^{*}-272$ & 13 & $272-372$ & 10 & $372-512$ & 10 & 33 \\
\hline 3 / HDL LV-19 & TA* - 254 & 12 & $254-355$ & 6 & $355-477$ & 11 & 29 \\
\hline
\end{tabular}

TA: Temperatura ambiente 


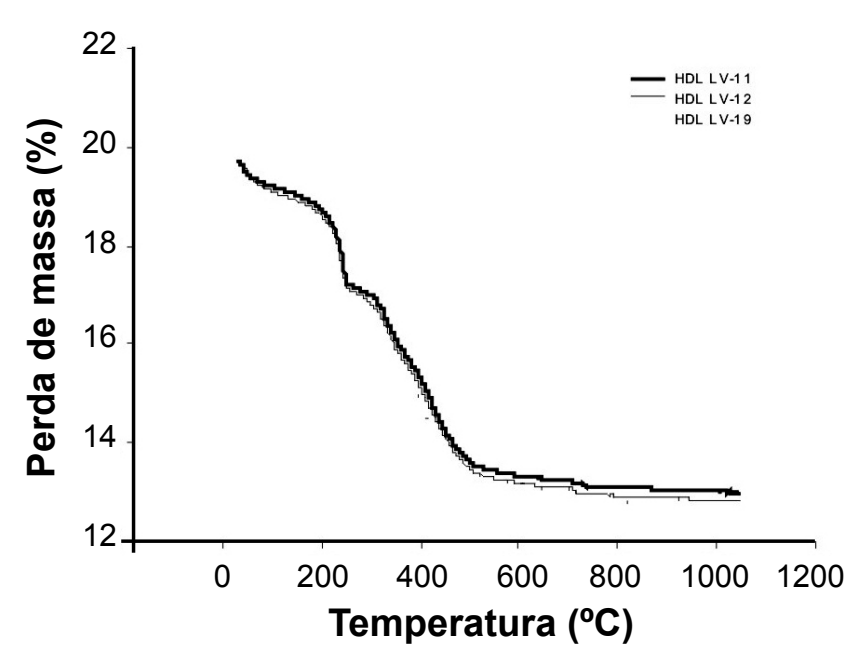

Figura 9: Curvas de análise termogravimétrica das amostras HDL LV-11, HDL LV-12 e HDL LV-19.

[Figure 9: TG curves of samples HDL-LV 11, LV-12 HDL and HDL LV-19.]

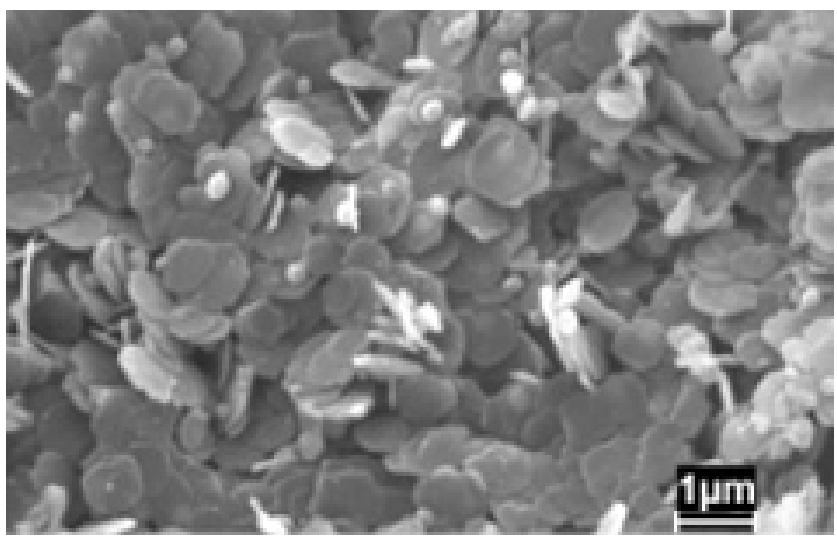

Figura 10: Imagem de microscopia eletrônica de varredura do composto HDL LV-20.

[Figure 10: SEM image of the compound HDL LV-20.]

\section{CONCLUSÕES}

Nas condições utilizadas neste trabalho foi possível realizar a síntese de HDLs a partir da lama vermelha. Os HDLs sintetizados pelo método de coprecipitação a $\mathrm{pH}$ variável (decrescente) apresentaram boa cristalinidade, parâmetros da célula unitária a e c e valores de espaçamento basal similares aos encontrados na literatura. Os resultados das análises de difração de raios X sugerem que o aumento do tempo do banho hidrotérmico favorece a cristalinidade dos compostos; entretanto, propiciam também a formação da hematita. Os compostos que foram submetidos aos tempos de 30 e 60 dias de tratamento hidrotérmico apresentaram uma melhor cristalinidade. Não foi observada nenhuma influencia do tempo de gotejamento estudado na cristalinidade e no tamanho de compostos sintetizados. Através da análise de espectroscopia na região do infravermelho foi possível identificar o ânion carbonato incorporado na estrutura dos materiais. Os resultados das análises, termogravimétrica e térmica diferencial, mostraram que a decomposição térmica ocorre em três etapas, com uma perda de massa total de aproximadamente $30 \%$. Os resultados de microscopia eletrônica de varredura apresentaram micro-grafias com dimensões nanométricas $(<0,2 \mu \mathrm{m})$. Além disso, foi observado que os compostos que apresentaram os cristais mais bem desenvolvidos foram aqueles submetidos há maiores tempo de banho hidrotérmico.

\section{REFERÊNCIAS}

[1] E. L. Crepaldi, J. B. Valim, Química Nova 21 (1998) 3.

[2] L. P. Cardoso, "Estudo da remoção de compostos orgânicos derivados da produção de poliéster presentes em efluentes industriais, por meio de sorção em hidróxidos duplos lamelares do sistema Mg-Al-CO,", Diss. Mestrado, USP, S. Paulo, SP (2002).

[3] A. de Roy, C. Forano, K. El Malki, J. P. Besse, “Anionic clays: trends in pillaring chemistry", M. L. Occeli, H. E. Robson, Synthesis of Microporous Materials, Van Nostrand Reinhold, New York, EUA (1992) cap. 7.

[4] W. T. Reichle, Solid State Ionics 22 (1986) 135.

[5] K. Kuma, W. Paplawsky, B. Gedulin, G. Arrhenius, "Mixed-valence hydroxides as bio-organic host minerals", $9^{\text {th }}$ Int. Conf. Origins of Life, Praga, Tchecoslováquia (1989).

[6] S. Miyata, T. Kumura, Chem. Lett 2, 8 (1973) 843.

[7] N. W. Menzies, I. M. Fulton, W. J. Morrell, J. Environ. Qual. 33 (2004) 1877.

[8] D. Donaire, "Gestão ambiental na empresa", Atlas, S. Paulo, SP (1995).

[9] Associação Brasileira do Alumínio, http://www. abal. org.br/ conheca/html/\#link1, acessada em 01/2008.

[10] Alunorte, http://www.vale.com/vale/cgi/cgilua.exe/sys/ start.htm?sid=566, acessada em 05/2008.

[11] M. G. Sujana, R. S. Thakur, B. C. Acharya, S. N. Das, S. B. Rao, "Effect of calcination and physico-chemical properties of red mud", Light Metals (1996) 93-98.

[12] G. Douglas, B. Degens, "A synopsis of potential amendments and techniques for the neutralization of acidic drainage waters in the Western Australian Wheatbelt", CSIRO Land Water Sci. Report 46 (2006) 6.

[13] M. Fahey, T. A. Newson, Y. Fujiyasu, "Engineering with tailings", in Environmental Geotechnics, Rio de Janeiro, RJ (2002).

[14] I. Doye, J. Duchesne, Appl. Geochem. 18 (2003) 1197. [16] L. Santona, P. Castaldi, P. Melis, J. Hazardous Mater. 136, 2 (2006) 324.

[17] J. Halász, M. Hodos, I. Hannus, G. Tasi, I. Kiricsi, "Catalytic detoxification of C2-chlorohydrocarbons over iron-containing oxide and zeolite catalysts", Colloids and Surfaces A: Physicochem. Eng. Aspects 265 (2005) 171.

[18] S. Ordóñez, H. Sastre, F. V. Díez, "Hydrodechlorination of tetrachloroethylene over sulfided catalysts: kinetic study", Catalysis Today 73 (2002) 325.

[19] H. Genç, J. C. Tjell, D. McConchie, O. Schuiling, "Adsorption of arsenate from water using neutralized red 
mud", J. Colloid and Interface Sci. 264 (2003) 327.

[20] H. Genç-Fuhrman, H. Bregnhoj, D. McConchie, "Arsenate removal from water using sand-red mud columns", Water Res. 39 (2005) 2944.

[21] H. Genç-Fuhrman, J. C. Tjell, D. McConchie, "Increasing the arsenate adsorption capacity of neutralized red mud (Baouxsol)", J. Colloid Interface Sci. 271 (2004) 313.

[22] R. Apak, K. Gluçu, M. H. Turgut, "Modeling of Cooper (II), Cadmium (II) and Lead (II) adsorption on red mud", J. Colloid Interface Sci. 203 (1998a) 122.

[23] R. Apak, E. Tutem, M. Hugul, E. Hival, "Heavy metal cation retention by unconventional sorbents: red mud and fly ashes", Water Res. 39 (1998b) 2.

[24] V. K. Gupta, M. Gupta, S. Shama, "Process developed for removal of lead and chromium from aqueous using red mud: an aluminium industry waste", Water Res. 35 (2001) 5. [25] E. B. Silva Filho, W. Brander Junior, V. L. Silva, M. A. Motta Sobrinho, M. C. M. Alves, "Tratamento de efluentes têxteis por adsorção em lama vermelha", $23^{\circ}$ Cong. Bras. Eng. Sanit. Amb., Campo Grande, Mato Grosso (2005).

[26] Y. Çengeloglu, E. Kir, M. Ersöz, "Recovery and Concentration of $\mathrm{Al}(\mathrm{III}), \mathrm{Fe}(\mathrm{III}), \mathrm{Ti}(\mathrm{IV})$, and $\mathrm{Na}$ (I) from Red Mud”, J. Colloid Interface Sci. 244 (2001) 342.

[27] C. Brunori, C. Cremisini, P. Massanisso, V. Pinto, L. Torricelli, "Reuse of a treated red mud bauxite waste: studies on environmental compatibility", J. Hazardous Mater. 117 (2005) 1 .

[28] G. Akay, B. Keskinler, A. Çakici, U. Danis, "Phosphate
Removal from Water by Red Mud using Crossflow Microfiltration”, Water Res. 32 (1998) 3.

[29] E. Lopez, B. Soto, M. Arias, A. Nunez, D. Rubinos, M. T. Barral, "Adsorbent properties of red mud and its use for wastewater treatment", Water Res. 32 (1998) 4.

[30] G. E. Ho, R. A. Gibbs, K. Methew, "Bacteria and virus removal from secondary effluent in sand and red mud columns”, Water Res. 26 (1991) 261.

[31] G. M. P. De Sousa, “Avaliação do Sistema de Tratamento do Chorume Utilizado no Aterro Sanitário do Aurá: Proposta de Utilização da Lama Vermelha como Adsorvente da Matéria Orgânica Presente no Chrorume", Diss. Mestrado, UFPa, PR (2000).

[32] M. P. G. De Souza, L. B. C. Menezes, C. A. G. De Souza, R. F. Neves, "Utilização de lama vermelha como adsorventes da matéria orgânica presente no chorume (Aterro Sanitário do Aurá, Belém, Pará)", 13 Cong. Bras. Eng. Química, Águas de S. Pedro, SP (2001).

[33] D. B. Kirkpatrick, "Red mud product development", The Minerals, Metals Mater. Soc. (1996).

[34] D. McConchie, M. Clark, F. Davies-McConchie, "New strategies for the management of bauxite refinery residues (red mud)", Proc. 6 ${ }^{\text {th }}$ Int. Alumina Quality Workshop, Brisbane, Australia (2002).

[35] R. N. Summers, M. R. Rivers, M. F. Clarke, "The use of bauxite residue to control diffuse phosphorus pollution in Western Australia: a win-win-win outcome", $6^{\text {th }}$ Int. Alumina Quality Workshop, Brisbane, Austrália (2002).

(Rec. 20/10/2009, Rev. 16/11/2009, Ac. 20/05/2010) 